Revue européenne des sciences sociales

European Journal of Social Sciences

XLVIII-145 | 2010

À la recherche des fondements de la rationalité

\title{
Les sociologues et le postulat de rationalité
}

\section{Jacques Coenen-Huther}

\section{OpenEdition}

\section{Journals}

Édition électronique

URL : http://journals.openedition.org/ress/735

DOI : $10.4000 /$ ress.735

ISSN : 1663-4446

\section{Éditeur}

Librairie Droz

\section{Édition imprimée}

Date de publication : 1 mars 2010

Pagination : 5-16

ISBN : 978-2-600-01427-4

ISSN : 0048-8046

Référence électronique

Jacques Coenen-Huther, "Les sociologues et le postulat de rationalité », Revue européenne des sciences sociales [En ligne], XLVIII-145 | 2010, mis en ligne le 01 mars 2013, consulté le 30 avril 2019. URL : http://journals.openedition.org/ress/735; DOI : 10.4000/ress.735 
Jacques COENEN-HUTHER

\section{LES SOCIOLOGUES ET LE POSTULAT DE RATIONALITÉ ${ }^{1}$}

Depuis longtemps, la notion de rationalité est un objet de préoccupations pour les sociologues, soit qu'ils visent à établir les critères de l'action rationnelle, soit qu'ils veuillent montrer le caractère illusoire de l'exercice. La tendance inhérente aux raisonnements sociologiques est de définir le comportement rationnel de manière nettement moins restrictive que les économistes ne sont tentés de le faire. Si la rationalité devait être identifiée à la rationalité instrumentale, orientée vers l'optimisation des avantages matériels, de nombreuses activités chargées de sens seraient exclues du champ de l'action rationnelle. La plupart des sociologues qui, à l'instar de Raymond Boudon, adhèrent à une conception cognitiviste, ou rationaliste, de l'analyse sociologique cherchent à définir le comportement rationnel par une adéquation entre les raisons d'agir des individus et leurs actions. Certes, ils s'écartent des théories rudimentaires du «choix rationnel» et conviennent que la rationalité est une notion relative: relative eu égard aux aspects formels de la situation et relative eu égard aux caractéristiques de l'acteur. Dans cette perspective, la motivation à agir peut être la promotion d'intérêts - même s'ils ne sont pas toujours de nature économique - ou, au contraire, le respect désintéressé d'une valeur. Le comportement mû par une valeur peut être tenu pour digne d'éloges mais inadéquat pour la protection des intérêts légitimes de l'acteur.

Il n'est cependant pas certain que tous les adeptes de la sociologie cognitiviste soient pleinement conscients des implications de cette relativité intrinsèque de la notion de rationalité. Elle signifie en effet que plus une société est socialement et culturellement hétérogène, moins on peut s'attendre à y trouver un consensus sur les critères du comportement rationnel. On n'en veut pour preuve que l'habitude solidement ancrée de faire de l'adjectif «irrationnel» un terme désobligeant pour disqualifier une manière de se comporter suscitant le dédain ou la désapprobation. La rationalité doit être mise en rapport avec le contexte socioculturel. D'une période de l'histoire à une autre, d'une culture à une autre, la notion de comportement rationnel acceptée par le sens commun peut varier énormément. Il n'est, par exemple, nullement déraisonnable de lier le processus de rationalisation discerné par Max Weber à une conception de la rationalité émanant du monde occidental.

1 Une première version, plus brève, de cet article a paru en anglais sous le titre The Postulate of Rationality between Universality and Diversity (Coenen-Huther, 2009). 


\section{RATIONALITÉ OU COHÉRENCE?}

Dans ces conditions, c'est l'intérêt même de l'attribution de rationalité qui peut offrir matière à discussions. Est-ce tellement important pour un sociologue de décider si une ligne de conduite particulière est rationnelle ou non? La réponse réside dans la relation qu'il entretient avec l'objet de sa recherche. Décider de la rationalité d'une action n'est pas seulement affaire de vérification empirique, c'est aussi - et même surtout - une question de présuppositions. Le sociologue peut être enclin à postuler la rationalité pour comprendre le comportement de l'acteur. De fait, l'idée de rationalité est en relation étroite avec la compréhension sociologique au sens du Verstehen weberien. Et comme les raisonnements sociologiques incorporent nolens volens une tendance au relativisme, on préserve la validité du postulat en distinguant plusieurs sortes de rationalités: rationalité instrumentale, rationalité axiologique, rationalité cognitive, rationalité limitée, etc. Dès lors, on peut se demander si l'acteur n'est pas censé manifester une certaine forme de rationalité parce que le sociologue se donne pour tâche d'analyser son comportement de façon rationnelle. La rationalité présumée de l'acteur refléterait alors le rationalisme du sociologue. En d'autres termes, l'observateur présupposerait un type de rationalité qu'il puisse endosser parce que cela semblerait le seul moyen de rendre intelligible à ses yeux le comportement de l'individu observé. Mais faut-il adhérer à une version élaborée de la distinction paretienne entre actions «logiques» et «non logiques»? Ne pourrait-on défendre l'idée que les prétendues «actions non logiques» ont leur logique propre que seul un point de vue tout à fait particulier permet de qualifier de «non logique»?

Le caractère éminemment relatif de la rationalité pourrait être la raison de se donner un concept de portée plus large qui serait un concept de cohérence. On substituerait ainsi un postulat de cohérence au postulat de rationalité. Un comportement peut être complètement irrationnel du point de vue de l'observateur extérieur mais n'en avoir pas moins une incontestable cohérence interne. Il peut trouver son origine dans un état émotionnel qu'il n'est guère possible de traduire en raisons bien élaborées, mais donner lieu quand même à une séquence comportementale parfaitement cohérente, impliquant une relation logique entre buts et moyens. La cohérence peut donc être admise, quand bien même l'idée de rationalité semble dénuée de pertinence. Le métier de sociologue consiste précisément à mettre en évidence une certaine logique là où le sens commun ne voit qu'absurdité. Si le comportement en question apparaît totalement incohérent - dans le cas d'une maladie mentale, par exemple - il échappe tout simplement à l'analyse sociologique dont il est hors de portée.

La compréhension rationnelle du comportement d'un individu n'est rien d'autre que la reconstruction hypothétique de sa logique d'action, basée sur des raisons qui paraissent aller de soi au sociologue. Il arrive que ces raisons coïncident avec les motivations ou les justifications présentées par l'acteur lui-même, mais c'est loin d'être toujours le cas. Comme la psychanalyse, mais de manière moins chargée de risques, l'analyse sociologique peut révéler l'individu à luimême. Quoi qu'il en soit, des raisons d'agir qui semblent évidentes au chercheur sont projetées sur le comportement de l'acteur. Il s'agit moins de faits observés ou 
d'informations récoltées que de raisonnements interprétatifs. Les données disponibles doivent être interprétées pour acquérir une signification. Ce processus analytique requiert plusieurs préalables hypothétiques. On présuppose tout d'abord l'existence de constantes de l'esprit humain qui transcendent les contextes sociaux, les ensembles culturels, voire les périodes de l'histoire. La réalité de telles constantes est niée de nos jours par les partisans d'un constructionnisme radical, mais c'est la condition sine qua non de toute étude scientifique de la vie sociale. Pour qu'un sociologue vivant à notre époque puisse comprendre une expérience religieuse datant de la Rome antique, il doit nécessairement y avoir de telles constantes. En second lieu - et corollairement à cette première présupposition -, le transfert à l'acteur de raisons d'agir conçues par le sociologue implique l'acceptation de la distinction simmelienne entre formes et contenu d'un cours d'action. Après avoir fourni l'interprétation de comportements observés dans le cadre d'un parti politique, on doit pouvoir séparer analytiquement leurs aspects formels de leur contenu, afin de pouvoir appliquer les leçons de l'analyse à un autre contexte - une institution religieuse, par exemple - et mettre en évidence une cohérence d'ordre général, sous-jacente à la diversité des actions envisagées dans leur contexte initial. Enfin, troisièmement, la compréhension sociologique présuppose une continuité entre la connaissance ordinaire et la connaissance sociologique, celle-ci étant dérivée de celle-là en raison d'une communauté de perceptions élémentaires. Le sociologue attribue à l'acteur des raisons d'agir qu'il juge lui-même convaincantes.

Cette conviction intime d'avoir atteint un niveau de compréhension libre de toute subjectivité ne s'atteint pas dans un vacuum social; elle s'appuie sur des intuitions que nul ne remet en question dans le monde de la vie quotidienne. Pour que les fondements logiques d'une argumentation s'imposent comme allant de soi, il faut qu'ils soient à ce point ancrées dans le sens commun qu'ils suscitent une impression d'universalité. Cette troisième condition de la compréhension sociologique exige une attitude intellectuelle très particulière. On ne peut que se fonder sur des évidences du sens commun, mais, en même temps, on doit s'employer à neutraliser mentalement la distance sociale et culturelle qui fait obstacle à l'acceptation de ces évidences. L'exercice est périlleux et il ne faut pas se dissimuler que l'étape du Verstehen, bien qu'inévitable, reste le maillon faible de toute argumentation sociologique. Le risque est grand de juger d'application universelle des conclusions qui ne sont valables que pour un certain milieu, à une certaine époque. Les chercheurs pratiquant l'observation participante sont généralement très conscients du caractère précaire de la compréhension sociologique et le compte rendu d'une recherche fondée sur l'observation directe est souvent le récit de faux départs, de malentendus et d'erreurs de jugement rectifiés à un stade plus avancé du travail de terrain ${ }^{2}$. Le sentiment d'évidence qui fonde la compréhension rationnelle est fortement soumis à l'air du temps. L'exemple classique, emprunté à Jaspers, de la mère giflant son enfant l'illustre à suffisance. Il y a vingt-cinq ans, la gifle était considérée comme une technique éducative tout à fait

2 J'ai développé ceci à l'aide d'exemples extraits de mes propres travaux de terrain dans un article publié antérieurement (Coenen-Huther, 1998). 
admissible et présentée comme un exemple d'action rationnelle (Boudon et Bourricaud, 1982, p. 3). Une décennie plus tard, elle n'était plus tolérée dans les milieux éduqués et apparaissait dans les raisonnements sociologiques comme un exemple type de comportement irrationnel (Valade, 1996, p. 545).

Dans la conception rationaliste, l'acteur est censé être mû par des raisons et ces raisons sont les variables indépendantes qui servent à expliquer son comportement. Néanmoins, comme c'est toujours le cas en analyse causale, l'accent placé sur une catégorie particulière de variables - en l'occurrence des raisons jugées plausibles - dépend d'un choix intellectuel qui constitue un préalable indispensable si l'on veut éviter la régression ad infinitum d'une cause à une autre. Dans l'éventualité d'un autre choix, si l'explication ne s'en tenait pas aux raisons attribuées à l'acteur, ces raisons cesseraient d'être des variables indépendantes et ne seraient plus que des variables intermédiaires dans la séquence de recherche. L'acteur n'en aurait pas moins de bonnes raisons d'opter pour une certaine ligne de conduite, mais il faudrait rendre compte de l'existence même de ces raisons qui n'auraient plus le statut de données. En amont des raisons attribuées à l'acteur, un élément d'une autre nature contribuerait à les expliquer et, de ce fait, à compléter l'analyse. Cette nouvelle catégorie explicative pourrait être composée de normes prédéfinies, exerçant leur pression sur l'individu. Il pourrait également s'agir de tout un processus de socialisation, incluant une variété d'agents socialisateurs ainsi qu'une gamme de moyens d'influence. Attribuer un rôle décisif à la socialisation et aux normes sociales, c'est sortir du cadre habituel de l'explication rationaliste. L'individu n'est plus l'être de raison né adulte. Le postulat de rationalité perd alors de son efficacité et l'opposition rationnel-irrationnel n'a plus la même vertu heuristique. C'est alors qu'intervient le postulat de cohérence qui relance l'analyse sociologique sur de nouvelles bases.

En soi, l'étude des processus de socialisation offre un vaste champ d'investigations en fournissant des hypothèses utiles sur l'impact comparatif des différentes normes et des sanctions qui leur sont associées. Le comportement d'un individu s'explique en partie par le succès ou l'échec de stratégies de socialisation. Au cours du dernier quart de siècle, on a accordé beaucoup d'attention aux conséquences des actions socialisatrices mises en œuvre dans les relations parents-enfants. La recherche empirique a montré que différentes combinaisons de contrôle et de soutien conduisent à des attitudes contrastées à l'issue du processus de socialisation (Peterson et Boyd, 1987). A première vue, substituer les normes sociales aux raisons individuelles comme variables explicatives peut avoir pour effet d'exagérer l'importance des contraintes collectives et de renforcer inutilement une conception holiste du social. Ce serait le cas si l'étude de la vie sociale était inévitablement confrontée à l'alternative holisme-individualisme. Mais une conception relationnelle du social supprime ce dilemme factice. Selon cette option, la relation interindividuelle - ou, si l'on préfère, le rapport social devient l'unité de la théorie sociologique et la société apparaît comme un réseau complexe d'interactions plus ou moins institutionnalisées. Chaque rapport social comporte un potentiel variable de contrainte et de liberté dû à l'asymétrie des relations. Les participants sont soumis à des normes de comportement mais sont également producteurs de normes en raison même du caractère récurrent de leurs 
contacts; l'individu et la société sont ainsi mutuellement transcendants. En dépit des pesanteurs sociales, la double contingence présente dans chaque interaction fait ressurgir l'aspect délibératif du comportement humain.

\section{LA COHÉRENCE DANS UNE SOCIÉTÉ DIVERSIFIÉE}

Un postulat de cohérence est l'instrument indispensable de l'analyse sociologique: rien de plus mais rien de moins. Ce postulat est de portée plus large que l'attribution de rationalité qui n'a pas les mêmes conséquences pratiques. Plus que l'insaisissable rationalité, la cohérence est une variable opératoire de la recherche de terrain. Le degré de cohérence est l'objet de travaux empiriques qui peuvent bénéficier des avancées de la théorie des rôles sociaux. Il est assez commun de conceptualiser l'homo sociologicus comme un individu porteur de rôles, réagissant à des attentes de rôles qui résultent d'influences normatives. Dans cette optique, le degré de cohérence d'une conduite est en grande partie la conséquence de caractéristiques structurelles de la société prise comme un tout. Dans une société plus ou moins homogène, fondée sur une division du travail très limitée, les rôles se superposent et une mesure de cohérence s'impose d'une certaine façon à l'individu. A l'inverse, dans un environnement social très diversifié, l'individu est confronté à une variété d'attentes de rôles qui ne favorisent pas une cohérence comportementale d'ensemble. Adoptant les termes de Merton, on dira que l'individu est caractérisé par une pluralité de statuts - le status set - et qu'à chacun de ces statuts est associée une série de rôles - le role set-correspondant aux attentes diverses des personnes concernées (Merton, 1957, pp. 110-111). Le concept de role set implique non seulement une pluralité de statuts et de rôles, mais aussi la possibilité de faire face à des attentes de rôles et des contraintes normatives qui peuvent être parfois tout à fait contradictoires. Selon Merton, «moins une société est intégrée, plus souvent l'individu sera soumis à la pression de rôles sociaux incompatibles» qui ne peuvent faire de lui qu'une «personne au soi multiple» (1949, 1968, p. 170). Le concept de rôle social réconcilie l'apparente incohérence de comportements insérés dans des contextes divers avec la cohérence d'ensemble du «soi multiple».

De toute évidence, quand un acteur se déplace d'un contexte social à un autre, les occasions de qualifier son comportement d'irrationnel se multiplient. Pour reprendre un des exemples évoqués par Merton, les actions de «l'homme marginal» subissant les pressions de sociétés aux exigences contradictoires seront certainement qualifiées d'irrationnelles à l'un ou l'autre moment (ibid.), mais l'apparence d'irrationalité peut très bien résulter d'une confrontation entre des attitudes inspirée par la rationalité instrumentale et d'autres, dictées par une forme de rationalité axiologique. Il faut cependant garder à l'esprit qu'une telle analyse n'est concevable que si le sociologue postule «derrière les raisons » des normes. En d'autres termes, la cohérence d'un comportement ne peut s'apprécier qu'en dotant l'individu de repères sociaux.

Les variables de configuration, les fameuses pattern-variables parsoniennes, permettent de nombreuses illustrations de l'interrelation entre l'acteur et le 
système social constitué par son environnement. Pour se référer à un exemple classique, l'enseignant dont le fils est un élève de sa classe est censé traiter celuici de deux façons différentes selon le rôle qu'il adopte. En tant qu'enseignant, il doit se comporter à l'égard de son fils comme à l'égard de n'importe quel autre élève, c'est-à-dire selon des normes de comportement à caractère universaliste, spécifique, affectivement neutre et centré sur la performance. Comme père cependant, bien qu'il ait à se préoccuper des accomplissements de son enfant, il convient qu'il le traite selon des normes pouvant être décrites comme particularistes, de portée diffuse et chargées d'affectivité. Dans nos sociétés modernes, on s'attend à ce que le «père-enseignant» se situe dans deux registres différents et présente ainsi deux catégories distinctes d'activités cohérentes. Les deux rôles qui le lient à son «fils-élève» ne peuvent se contaminer mutuellement. En serait-il ainsi que le comportement qui en résulterait serait considéré comme déviant. La modernité occidentale implique une segmentation des réseaux sociaux et requiert en conséquence une relative ségrégation des rôles. Ceci n'empêche qu'on puisse chercher à définir une cohérence surplombante. L'enseignant qui s'efforce d'appliquer des normes universalistes à l'école et des normes particularistes dans le cadre du foyer familial est sensible à la nécessité d'adopter ces deux manières de se comporter. En adoptant deux attitudes différentes selon le contexte, il s'adapte à la segmentation des réseaux caractéristique de son environnement social, mais il se soumet en même temps aux normes de comportement typiques de la société dans laquelle il vit. Le sentiment qui le guide est sans doute la conscience plus ou moins nette de la nécessité de «jouer le jeu selon les règles». Le contenu normatif de cette cohérence d'ensemble devient apparent quand un individu ne se comporte pas selon les règles et perturbe ainsi la vie sociale.

Dans une perspective sociologique, il est utile de se rappeler que dans d'autres sociétés que les nôtres, les règles généralement admises et la cohérence attendue sont différentes. La contamination de rôles spécifiques par des normes particularistes y est aisément acceptée et ne donne pas lieu à la réprobation morale. Ainsi, le comportement déviant d'une culture reste le comportement modal d'une autre culture, même si les Pyrénées ont cessé d'être la barrière la plus pertinente à cet égard. Le degré de cohérence d'un comportement individuel est induit en partie par les macro-structures contribuant à modeler l'environnement social. On ne peut exclure que les raisons par lesquelles un individu justifie sa conduite ne soient assez différentes des raisons qui lui sont attribuées par un observateur pratiquant la recherche sociologique dans un cadre peu familier. Même si ce dernier fait un effort pour s'immerger dans une culture autre que la sienne, son cadre de référence cognitif et normatif peut faire obstacle à une compréhension adéquate, à égale distance des deux extrêmes de l'adoption pure et simple des schèmes mentaux de l'individu observé - «going native » disent les anthropologues - et du recours à des catégories conceptuelles imposées de l'extérieur.

C'est d'un point de vue méthodologique et non ontologique qu'il s'impose de s'en tenir au principe selon lequel un comportement doit être tenu pour cohérent afin de pouvoir être analysé avec fruit. Cette présomption de cohérence ne peut cependant être séparée du choix préalable d'une perspective. Le jugement de cohérence est dépendant des limites assignées au système social pris en considé- 
ration. Face à une situation paraissant chaotique et à des actions laissant une impression d'absurdité, un jugement global d'incohérence n'a guère d'intérêt. Non qu'il ne puisse avoir un sens, philosophiquement parlant, mais il n'est ni pertinent ni fructueux pour la recherche sociologique. Rétrospectivement, certains phénomènes de grande ampleur qui dominèrent l'histoire du $20^{\mathrm{e}}$ siècle semblent marqués par un manque total de cohérence. Le génocide des Juifs opéré par l'Allemagne nazie avait, globalement, toutes les caractéristiques d'une entreprise complètement incohérente, particulièrement lorsqu'elle utilisait des ressources et de la main-d'œuvre qui auraient été nécessaires pour mener une guerre victorieuse. Cependant, isolée du contexte des opérations militaires, la mise en œuvre d'activités meurtrières à grande échelle fut organisée de façon très cohérente. On peut même constater qu'il y eut un progrès graduel dans l'organisation des assassinats de masse. Au surplus, au micro-niveau des actions individuelles, de nombreux acteurs impliqués dans l'entreprise d'extermination ont présenté des comportements parfaitement cohérents à des points de vue divers: hommes d'affaires bénéficiant de «l'aryanisation » des entreprises, fonctionnaires visant une promotion, membres du parti nazi croyant à la nécessité de débarrasser leur pays d'éléments allogènes, gardes des camps d'extermination cherchant à échapper au service armé dans les zones de batailles, etc. Ces tactiques individuelles ne furent pas à l'origine de la stratégie génocidaire mais elles lui imprimèrent sa dynamique.

Divers aspects de la politique de l'Union Soviétique appellent des constatations semblables. Le gouvernement par l'intimidation ou la terreur et le recours systématique au travail forcé ne pouvaient conduire en aucune façon à la société civile vigoureuse qu'exigeaient le développement économique de grande envergure et la très large démocratie préconisés par le régime lui-même. Néanmoins, ce sont précisément les aspects contradictoires du totalitarisme soviétique qui contribuèrent à l'apparition d'attitudes cohérentes de la part d'individus engagés dans des stratégies de survie. Dans son roman Une journée d'Ivan Denissovitch (1967), Alexandre Soljenitsyne décrit une séquence d'interaction parfaitement cohérente au cœur d'une situation qui l'est beaucoup moins. Les détenus d'un camp de travail sibérien doivent survivre par des températures de -30 à -35 degrés centigrades. Chaque jour, ils chapardent des petits morceaux de bois sur les chantiers et les ramènent au camp pour pallier le manque de combustible. Cette manière de se tirer d'affaire est évidemment interdite et le personnel de garde doit confisquer les maigres fagots ainsi rassemblés. Les gardes les confisquent d'autant plus volontiers qu'ils souffrent eux-mêmes du froid et agissent dans leur intérêt personnel. Il leur est cependant impossible d'appliquer strictement le règlement et de procéder à une confiscation totale car les détenus n'auraient alors plus aucune raison de ramasser du bois. La confiscation quotidienne ne peut donc être que partielle. On a affaire ici à des actions qui ne sont certainement pas cohérentes avec les normes établies par les autorités mais qui se déroulent de façon très cohérente au cours d'un processus d'interaction récurrent.

Envisageons à présent des politiques gouvernementales plus spécifiques. L'écart entre les buts visés et les résultats atteints peut très souvent donner une impression d'incohérence ou d'absurdité. Cette absence de cohérence peut néan- 
moins être la conséquence d'une action conçue logiquement mais engagée avec une connaissance insuffisante du contexte systémique. Dans de tels cas, rationalité et erreur de jugement ne sont pas nécessairement contradictoires (Bronner, 2008, p. 172). Des mesures gouvernementales récentes visant à combattre l'inflation en Chine en fournissent l'illustration. Les autorités chinoises ordonnèrent aux services publics de bloquer les prix de l'électricité. Le décret fut promulgué pour protéger la population de la montée des prix et pour permettre aux ménages d'équilibrer leur budget. Mais le contexte n'était plus celui d'une économie administrée et la décision prise eut très vite des conséquences inattendues. Les centrales électriques réagirent en réduisant leurs achats de charbon. Les charbonnages abaissèrent alors leur niveau de production. En raison des températures hivernales, la demande de charbon augmenta jusqu'à excéder la production et les centrales électriques se retrouvèrent avec des stocks très insuffisants. Il en résulta des coupures de courant et des fermetures d'usines (McDonald, 2008). Les autorités avaient réagi aux tendances inflationnistes par des mesures qui auraient été adéquates dans le contexte d'une économie planifiée mais qui n'étaient plus appropriées dans le cadre d'une économie déjà dominée par les mécanismes du marché.

\section{L'AMBITION SCIENTIFIQUE ET L'ÉVALUATION DE LA COHÉRENCE}

Compte tenu de son ambition scientifique, la sociologie ne peut que viser à l'universalité, ce qui signifie que le but ultime de la recherche sociologique est de formuler des propositions ayant une validité universelle. En conséquence, il y a une tension évidente entre les attributions de rationalité, voire même de cohérence, d'une part, et l'objectif scientifique de connaissance à portée universelle, d'autre part. Pour être acceptés comme des propositions à validité universelle, les diagnostics de cohérence doivent être posés dans un contexte social hypothétique de transsubjectivité complète. Un tel contexte implique que les motivations conscientes de l'acteur individuel - les raisons qu'il estime lui-même être au fondement de ses actions ou de ses croyances - sont largement perçues comme convaincantes dans son milieu social. La connaissance sociologique se greffe alors sur le sens commun dont elle rencontre les manières de penser et on peut, avec Leo Strauss (1953) lui conférer le statut de «connaissance dérivée ${ }^{3}$. Une situation de consensus général sur l'interprétation d'un comportement n'est imaginable qu'à partir de la présupposition implicite que tous les individus sont interchangeables et peuvent souscrire à la même argumentation. Toutefois, dès que le contexte social est hétérogène, l'aspect convaincant des raisons ou des croyances individuelles devient matière à discussions. Ceci est assez compréhensible si l'on tient compte du fait qu'elles ne sont «ni objectives ni purement subjectives» mais doivent être largement perçues comme convaincantes (Boudon, 1997, p. 53). Dans le langage de la vie quotidienne, les raisons de l'acteur individuel, telles qu'elles sont imaginées par d'autres, sont les «bonnes

3 On trouvera une illustration de ce caractère dérivé, fondée sur des observations de terrain, dans un article paru récemment dans cette revue (Coenen-Huther, 2007, p. 37-39). 
raisons» qu'il a d'agir comme il le fait. Pour que ces «bonnes raisons » puissent être considérées comme des «raisons fortes », rencontrant une approbation générale, elles doivent participer d'un mode de pensée typique de toute une population ou, au moins, d'un groupe relativement homogène.

Tant qu'on s'en tient au contexte hypothétique de transsubjectivité complète, les contacts avec nos contemporains renforcent les «bonnes raisons», quelle qu'ait pu être leur validité initiale. On doit néanmoins faire observer que ce processus a un caractère circulaire qui suscite le doute et invite à la réflexion. Une croyance, ou une raison d'agir d'une certaine façon, prend son sens pour l'acteur individuel quand elle s'appuie sur des «raisons fortes». Dans un environnement socialement et culturellement homogène, l'acteur se rend compte que ces raisons sont largement partagées autour de lui et s'insèrent dans des argumentations quasiment stéréotypées. Nulle voix dissidente ne défend des raisonnements concurrents, pouvant également s'ancrer dans des « raisons fortes ». Le consensus relatif à une croyance ou une raison d'agir ne peut manquer d'avoir un impact sur les esprits. L'acteur individuel sent que ses raisons sont fortes parce que chacun autour de lui se déclare d'accord. Et chacun se déclare d'accord parce que ces raisons sont perçues comme fortes. Il est vrai qu'il n'y a probablement pas moyen d'échapper à de telles tautologies parce qu'elles sont, presque par définition, au cœur de la vie sociale. Dans toute situation d'interaction, des individus s'influencent les uns les autres et cette influence mutuelle a des conséquences pour d'autres acteurs. Autrement dit, des normes de comportement émergent d'une agrégation de raisons ou de croyances individuelles. Mais ce phénomène émergent devient autonome en ce sens que les normes produites, à leur tour, stimulent des croyances individuelles. Jusqu'à ce que de nouvelles occasions d'interaction les mettent à l'épreuve...

Dans la réalité empirique de sociétés diversifiées et stratifiées, les interactions peuvent être de nature extrêmement différente selon l'environnement social ou culturel. La transsubjectivité suscitant un sentiment d'évidence est généralement limitée à un certain milieu social, bien que la plupart des acteurs ne s'en rendent pas compte. Il est donc permis de douter que la notion hypothétique d'entière transsubjectivité suffise à faire accéder des propositions sociologiques à l'universalité ${ }^{4}$. Dès lors, le mécanisme cognitif le plus pertinent - qui relègue la transsubjectivité au rang de variable dépendante - est le processus intersubjectif par lequel les individus ajustent leur manière de penser au cours de séquences d'interaction réelles (Chazel, 1997, p. 205). Ces ajustements sont d'autant plus aisés que les individus entretiennent des contacts significatifs sur une base fréquente (ibid.). Outre l'analyse de situations de transsubjectivité hypothétique au cours desquelles les individus manifestent leur accord parce qu'ils raisonnent tous de la même façon, il existe un autre type de recherches visant à étudier les manières de raisonner typiques d'une catégorie sociale particulière. Elles apparaissent ainsi comme des signes d'appartenance: appartenance à un réseau social, à un groupe,

4 J'ai eu l'occasion de présenter ce raisonnement plus en détail, à partir d'autres prémisses, dans L'Année sociologique (Coenen-Huther, 2001). 
à une communauté. C'est dans les études de stratification sociale que cette démarche peut revêtir le plus d'intérêt. Lorsque des individus partagent certaines manières de penser, certaines croyances, certains espoirs, une classification objective basée sur des données statistiques recouvre un sentiment intersubjectif d'appartenance qui est le propre des classes sociales. L'intersubjectivité ancrée dans la réalité empirique se substitue alors aux manifestations idéal-typiques de transsubjectivité illustrées par des exemples construits.

\section{INTERSUBJECTIVITÉ ET COHÉRENCE}

Il paraît difficile d'examiner les mécanismes intersubjectifs présents dans les rapports sociaux sans recourir à la notion de socialisation évoquée plus haut. Tout partenaire d'une relation interindividuelle y apporte un bagage socioculturel contribuant à définir la manière dont il perçoit la situation et s'y adapte. Ce terme de «bagage» renvoie métaphoriquement à l'identité sociale de l'acteur, telle qu'elle a évolué jusqu'au moment où il s'est engagé dans la relation. C'est la combinaison conceptuelle identité-rôles - une identité fondée sur une pluralité de rôles, passés et présents - qui permet d'apprécier la cohérence d'une personnalité, au-delà de la diversité, souvent trompeuse, des rôles et des attentes de rôles. De prime abord, la variété des situations et des contextes paraît priver chaque rapport social de toute pertinence pour les autres. Mais plusieurs situations d'interaction incorporant au moins un élément commun ne peuvent être totalement indépendantes les unes des autres. Chacune est influencée par l'existence des autres et toutes contribuent à modeler la personnalité des individus concernés. Nous sommes donc le produit de l'imbrication des rapports sociaux et non d'une raison immanente. De telles situations, affectant les dispositions d'agir ultérieures, débutent à la naissance lorsque les parents du nouveau-né exerçent leur influence au cours de la phase de socialisation primaire. Ce sont donc de véritables chaînes d'interactions qui requièrent de l'individu un comportement adaptatif, même si cette adaptation consiste paradoxalement en une rébellion ou une fuite. Car les «bagages » sont loin d'être toujours du même poids. Tous les individus ne se trouvent pas confrontés aux mêmes opportunités d'interaction. Il en est qui n'auront jamais la possibilité de faire certaines expériences parce qu'elles sont socialement hors de leur portée. Malgré la diversification des hiérarchies sociales et la fluidité des affiliations, les différences de ressources entre les catégories sociales restent considérables. Le langage de la lutte des classes paraît aujourd'hui obsolète, mais il n'en subsiste pas moins des riches et des pauvres, des puissants et des faibles, des dominants et des dominés, les élites et la masse; ces dichotomies sommaires n’ont pas cessé de conserver une réalité objective et une signification subjective.

Ceci dit, quelle que soit la position sociale d'un individu et les dispositions personnelles qu'elle favorise, il y a une interpénétration permanente des composantes individuelles et sociales de la personnalité. C'est le résultat d'un mouvement de socialisation constant et c'est ce qui rend problématique une approche purement rationaliste du comportement humain. La cohérence s'évalue par rapport aux raisons d'agir de l'individu, mais aussi par rapport à ses émotions et aux influences socialisatrices qu'il a subies. La construction du «soi » est un 
processus jamais achevé qui rend la conception dynamique héritée du pragmatisme américain sociologiquement plus pertinente que la vision cartésienne classique de la nature humaine. L'intersubjectivité, au sens du pragmatisme, est le résultat d'ajustements cognitifs réguliers dans des situations d'interaction et sur la base de ces situations. La formation graduelle de la personnalité dans la dynamique des relations interpersonnelles a été décrite et analysée en détail par George Herbert Mead (1934). Selon lui, l'individu se construit lui-même dans ses contacts avec les autres, dans un dialogue incessant entre les composantes réflexives et adaptatives de sa personnalité. L'aspect réflexif de la personnalité, créant la possibilité d'une distance mentale à l'égard des autres et de soi-même, n'existe que par l'influence humanisante de l'environnement social. C'est un élément capital de la socialisation. Tout au long d'une trajectoire de vie, plusieurs catégories $d^{\prime}$ ' «autres significatifs» se succèdent et exercent des influences diverses, parfois contradictoires, sur l'individu. Ce sont les étapes successives de la socialisation secondaire par laquelle l'individu acquiert ses capacités subjectives et accumule son bagage socioculturel.

Les mécanismes de socialisation secondaire sont particulièrement apparents lorsque des gens qui se connaissent de longue date cessent de se voir pendant une longue période. L'émouvant essai d'Alfred Schütz (1945), The Homecomer - se référant au G.I. rentrant aux Etats-Unis après les combats de la Deuxième Guerre mondiale - illustre l'évolution produite de manière convaincante. L'individu qui revient chez lui après un long séjour à l'étranger dans des conditions inhabituelles fait une impression étrange sur ceux qui l'ont connu autrefois. Il semble être devenu une personne différente. Pendant son absence, il a fait de nouvelles expériences qui l'ont transformé. Comme on dit dans son entourage, «il n'est plus le même». Un tel processus d'éloignement est encore plus net dans le cas de l'immigrant qui a laissé des personnes de référence dans son pays d'origine et en a acquis de nouvelles qui participent à sa resocialisation dans le pays où il s'installe. Les doutes intimes liés à cette transformation d'identité sont typiques du marginal man - l'homme marginal - évoqué par Robert Park (1928). Bien sûr, les changements affectant le réseau de relations ne sont pas uniquement le résultat de la mobilité géographique ou sociale. Ils peuvent être suscités par tous les événements qui marquent la vie: mariage, divorce, maladie, retraite, deuil, etc. L'influence des « autres significatifs »- partenaires, personnes de référence - se manifeste de deux façons. Elle a de toute évidence un impact sur l'élément adaptatif de la personnalité en provoquant une forme de contrôle social intériorisé. Elle a toutefois également une influence indirecte sur la composante réflexive du «soi». Quand de nouveaux personnages entrent en scène, il est temps de faire face à de nouvelles attentes de rôle mais c'est aussi le moment de renouveler le débat intérieur sur la base de nouvelles interactions et de nouveaux arguments.

$* * *$

En tant que mécanismes sociaux, les attentes de rôle, les processus de socialisation, les pressions normatives sortent du cadre de référence de la rationalité mais ils n'impliquent aucun déterminisme. Dans les conjonctures les plus impérieuses, la réflexivité de l'esprit humain préserve une marge de choix. Comme 
Peter Berger (1963, p. 165) l'a fait observer, on a toujours la possibilité de sortir d'un rôle.

\section{Université de Genève}

\section{RÉFÉRENCES BIBLIOGRAPHIQUES}

Berger, Peter L., Invitation to Sociology, Harmondsworth, Penguin, 1963.

Boudon, Raymond, «L'explication cognitiviste des croyances collectives». In: R. Boudon, A. Bouvier et F. Chazel, Eds, Cognition et sciences sociales, Paris, Presses universitaires de France, 1997.

Boudon, Raymond et Bourricaud, François, Dictionnaire critique de la sociologie, Paris, Presses universitaires de France, 1982.

Bronner, Gérald, «L'apport d'une épistémologie cognitive à la démarche sociologique », Revue Européenne des Sciences Sociales, vol. 46 (140), 2008, pp. 155-174.

Chazel, François, «Les ajustements cognitifs dans les mobilisations collectives: questions ouvertes et hypothèses ». In: R. Boudon, A. Bouvier et F. Chazel, Eds, Cognition et sciences sociales, Paris, Presses universitaires de France, 1997.

Coenen-Huther, Jacques, «Formes et ambiguïtés de la compréhension en situation d'observation», Cahiers internationaux de Sociologie, Vol. CIV, 1998, pp. 77-96.

Coenen-Huther, Jacques, «Sens moral ou raisons fortes?», L’Année sociologique, vol. 51 (1), 2001, pp. 233-254.

Coenen-Huther, Jacques, «Classifications, typologies et rapport aux valeurs », Revue Européenne des Sciences Sociales, vol. 45 (138), 2007, pp. 27-40.

Coenen-Huther, Jacques, «The Postulate of Rationality between Universality and Diversity», in: Mohamed Cherkaoui et Peter Hamilton, Eds, Raymond Boudon. A Life in Sociology. Essays in Honour of Raymond Boudon, Vol. 2, Oxford, The Bardwell Press, 2009, pp. 111-124.

McDonald, Joe, «Cooling Inflation, Communist-style», International Herald Tribune, February 12, 2008.

Mead, George Herbert, Mind, Self, and Society. Chicago, University of Chicago Press, 1934.

Merton, Robert K., Social Theory and Social Structure, 1949; deuxième édition augmentée, New York, The Free Press, 1968.

Merton, Robert K., «The Role-Set: Problems in Sociological Theory », The British Journal of Sociology, Vol. 8, 1957, pp. 106-120.

Park, Robert E., «Migration and the Marginal Man», American Journal of Sociology, vol. 33, 1928, pp. 200-206

Peterson, Gary W. et Rollins Boyd C. «Parent-Child Socialization », in: Sussman M. B. et Steimetz S. K., Eds, Handbook of Marriage and the Family, New York, Plenum Press, 1987, p. 471-507.

Schütz, Alfred, «The Homecomer», American Journal of Sociology, vol. 50 (5), 1945, pp. 369-376.

Soljenitsyne, Alexandre, Une journée d'Ivan Denissovitch. Récit, traduit du russe, Paris, Julliard, 1967.

Strauss, Leo, Natural Right and History, Chicago, University of Chicago Press, 1953.

Valade, Bernard, Introduction aux sciences sociales, Paris, Presses universitaires de France, coll. «Premier cycle», 1996. 